\title{
SOME PROBLEMS CONNECTED WITH THE CALCULATION OF STOP LOSS PREMIUMS FOR LARGE PORTFOLIOS
}

\author{
FREDRIK ESSCHER \\ Stockholm
}

\section{SUMMARY ${ }^{1}$ )}

When experience is insufficient to permit a direct empirical determination of the premium rates of a Stop Loss Cover, we have to fall back upon mathematical models from the theory of probability-especially the collective theory of risk-and upon such assumptions as may be considered reasonable.

The paper deals with some problems connected with such calculations of Stop Loss premiums for a portfolio consisting of non-life insurances. The portfolio was so large that the values of the premium rates and other quantities required could be approximated by their limit values, obtained according to theory when the expected number of claims tends to infinity.

The calculations were based on the following assumptions.

Let $F(x, t)$ denote the probability that the total amount of claims paid during a given period of time is $\leqslant x$ when the expected number of claims during the same period increases from $o$ to $t$. The net premium $\Pi(x, t)$ for a Stop Loss reinsurance covering the amount by which the total amount of claims paid during this period may exceed $x$, is defined by the formula

$$
\Pi(x, t)=\int_{x}^{\infty}(z-x) d F(z, t)
$$

and the variance of the amount $(z-x)$ to be paid on account of the Stop Loss Cover, by the formula

$$
\sigma_{\pi}^{2}=\int_{x}^{\infty}(z-x)^{2} d F(z, t)-[\Pi(x, t)]^{2}
$$

1) The complete paper is published in Skandinavisk Aktuarietidskrift r 965 . 
As to the distribution function $F(x, t)$ it is assumed that

$$
F(x, t)=\sum_{n=0}^{\infty} P_{n}(t) V_{(x)}^{n}
$$

where

$P_{n}(t)$ is the probability that $n$ claims have occurred during the given period, when the expected number of claims increases from o to $t$,

$V(x)$ is the distribution function of the claims, giving the conditioned probability that the amount of a claim is $\leqslant x$ when it is known that a claim has occurred, and

$V^{n *}(x)$ is the $n^{\text {th }}$ convolution of the function $V(x)$ with itself. $V(x)$ is supposed to be normalized so that the mean $=\mathrm{I}$.

The function $P_{n}(t)$ is assumed to be defined by the formula

$$
P_{n}(t)=\int_{0}^{\infty} \frac{e^{-\lambda t}(\lambda t)^{n}}{n !} d U(\lambda)
$$

where $U(\lambda)$ is a distribution function with the mean $\mathrm{r}$.

On the above assumptions it can be shown that

$$
q(s)=\lim _{t \rightarrow \infty} \frac{\Pi(s t, t)}{t}=\int_{0}^{\infty}(v-s) d U(v)
$$

and

$$
\sigma_{q}^{2}=\lim _{t \rightarrow \infty} \frac{\sigma^{2} \pi}{t^{2}}=\int_{0}^{\infty}(v-s)^{2} d U(v)-[q(s)]^{2}
$$

In investigations of this kind, $U(s)$ is to be interpreted as the distribution function of the fluctuations in the basic probabilities from one period to another. About $U(s)$, however, we know very little. We can-in favourable cases-calculate up to the second moment of $U(s)$ but, as a rule, our experience is insufficient to give us any hints as to the behaviour of $U(s)$. We have to fall back upon assumptions. As the limit values $q(s)$ and $\sigma_{q}$ depend only on the function $U(s)$, it was considered important to get a numerical illustration of the effect of different assumptions. For this purpose, some values of $q(s)$ and $\sigma_{q}$ were computed with $U(s)$ 
defined according to six different expressions, one of which was the incomplete $\Gamma$-function

$$
U(s)=\frac{\mathrm{I}}{\Gamma(k)} \int^{\star k} e^{-z} z^{k-1} d z
$$

In the well known investigations by Ammeter this function has been used to describe the fluctuations in the basic probabilities.

The six expressions were chosen in such a way that in all cases

$$
\int_{0}^{\infty} s d U(s)=\mathrm{I} \text { and } \int_{0}^{\infty}(s-\mathrm{I})^{2} d U(s)=\mathrm{I} / k
$$

corresponding to mean and variance according to (3).

The calculations were performed for $s=$ I.O, I.I, I.2 . I.5 with $k=20$ and $k=40$, approximately corresponding to $k$-values found for industrial fire insurance and liability insurance in Sweden.

From tables given in the paper it is seen that the function $q(s)$ is strongly dependent on the value of $k$, which fact seems to be the more pronounced the larger the value of $s$.

For fixed values of $k$ and $s$, the differences between the $q(s)$ values corresponding to the different assumptions about $U(s)$ are surprisingly small for $s$-values not exceeding I.2-I.3. When measured in proportion to the $q(s)$-values, the differences become more important the larger the value of $s$. The differences, however, and the $q(s)$-values themselves, are small when compared with the values of the standard deviations $\sigma_{q}$, which for $s=$ I.O amount to I.5 -2.0 times the corresponding $q(s)$-values and for, e.g., $s=\mathrm{I} .3$ amount to 4-7 times the $q(s)$-values. The large values of $\sigma_{q}$ indicate the necessity of a heavy security-loading of the net premium rate $q(s)$. Determined as a ratio of the $\sigma_{q}$ 's, say $50 \%$, the loading in most cases will amount to several times the differences between the different $q(s)$-values, even in cases where these differences are large. For the calculation of Stop Loss premiums to be used in practice it seems, for this reason, to be of very little importance whether the one or the other reasonable assumption is made as to the function $U(s)$.- For practical reasons, the assumption according to formula (3) is used in the following investigations. 
The error made when using $q(s)$ instead of $\Pi(s t, t) / t$ for the calculation of the premium rate is defined by the difference

$$
\Delta(s, t)=\frac{\Pi(s t, t)}{t}-q(s)
$$

By means of the characteristic functions corresponding to $F(x, t), U(s)$ and $V(x)$, the difference $\Delta(s, t)$ can be expressed as an absolutely convergent integral. Developing the integral in a series running in powers of $\mathrm{I} / t$, we get for large $t$-values a good approximation of $\Delta(s, t)$ already by the first term in the development.

Calculations of this kind were made for $t=10.000,20.000$, 50.000 and 100.000 and for the same values of $s$ and $k$ as before. As to the function $V(x)$ an assumption was made corresponding to a very "dangerous" claim distribution.

From the numerical results it is found that the limit function $q(s)$ gives a fairly good approximation of $\Pi(s t, t) / t$ already for values of $t$ around ro.0oo when $k=20$ and around 20.000 when $k=40$, and that the values of $\Delta(s, t)$ are quite negligible for $t$-values larger than 50.000. With less dangerous claim distributions than the one used in these calculations, corresponding results will be obtained even for more moderate values of $t$.

A large portfolio includes as a rule different groups of insurances, e.g. different insurance branches, some of which may be independent of each other in the sense that there is no co-variation between the fluctuations of the basic probabilities. This being so the distribution function of the total amount of claims for the entire portfolio is to be considered as the result of convolutions of the corresponding distribution functions for the different groups.

Formulae are derived permitting numerical calculations of $q(s)$ and $\sigma_{q}$ for such an amalgamated portfolio on the assumption that every group is so large that the premium rates-had the group been treated separately-could be approximated by their limit values. With three different assumptions about the composition of the portfolio, the values of $q(s)$ and $\sigma_{q}$ were calculated for $k=40$ and compared with the corresponding values for an undivided portfolio.

The results illustrate the very strong counterbalancing effect produced by an amalgamation of independent groups. If the port- 
folio consists e.g. of two independent groups of equal size, the $q(s)$-value for $s=\mathrm{I} .2$ will be only about a fourth and for $s=\mathrm{I} .3$ less than a tenth of the corresponding values for an undivided portfolio.

Also the $\sigma_{Q}$-values are strongly reduced by the amalgamations, although not in the same proportion as the $q(s)$-values.

The figures also illustrate the advantage of using a Stop Loss Cover embracing all the independent groups under one and the same treaty instead of having separate Covers for different groups. 